\title{
$N$-Phase Synchronization of Asymmetric Attractors in a Ring of Coupled Chaotic Circuits
}

\author{
Takuya Nishimoto*, Yoko Uwate*, Yasuteru Hosokawa ${ }^{\dagger}$, Yoshifumi Nishio* and Daniéle Fournier-Prunaret ${ }^{\ddagger}$ \\ *Dept. of Electrical and Electronic Eng., Tokushima University, \\ 2-1 Minami-Josanjima, Tokushima, 770-8506 Japan \\ Email: \{nishimoto, uwate, nishio\}@ee.tokushima-u.ac.jp \\ ${ }^{\dagger}$ Dept. of Media and Information Systems, Shikoku University, \\ Furukawa, Ohjin, Tokushima, 771-1192, Japan \\ Email: hosokawa@keiei.shikoku-u.ac.jp \\ ${ }^{\ddagger}$ LAAS-CNRS, 7 avenue du colonel Roche, F-31077 Toulouse Cedex 4, France \\ Email: daniele.fournier@insa-toulouse.fr
}

\begin{abstract}
Chaos synchronization can be observed in various natural systems. In this study, we investigate synchronization phenomena on a ring of coupled chaotic circuits. We can observe $N$-phase chaos synchronization and simple synchronization phenomena depending on initial values in the system. This paper suggests ring topology and alternately shifted bias are essential factors of $N$-phase synchronization.
\end{abstract}

\section{INTRODUCTION}

Chaos synchronization observed on large-scale coupled chaotic systems have been attracted attention in various science field because it can be regarded as models of real physical system. For instance, chaos phenomena have been reported in engineering, biology, economics, astronomy and so on. Therefore the investigation of synchronization phenomena on highorder chaotic systems are very important to grasp essentials of the phenomena observed in natural system.

On the other hand, some chaotic circuits have coexisting attractors [1]. Asymmetric attractor observed in the system which is symmetric structure causes some interesting phenomena [2],[3]. However there are not many studies of synchronization on asymmetric attractor. It is considered that observation of synchronization phenomena in the state of chaos on asymmetric attractor is difficult.

In this study, we also investigate synchronization phenomena in a ring of coupled chaotic system whose subcircuits generate asymmetric attractor. 2-types synchronization phenomena depending on initial values can be stably observed in the system. Especially, $N$-phase chaos synchronization observed in the system $(N=6 \sim 50)$ is extremely interesting phenomena since $N$-phase synchronization can be observed in small-scale coupled chaotic system normally. In this study, we confirm $N$-phase synchronization phenomena on large-scale system. Moreover, similar behaviour can be observed in another system with VDP circuit which include ideal current source. By carrying out circuit experiments and computer simulations, we can confirm the phenomena above. Namely, the phenomena possibly observed in many systems which have asymmetric attractor and ring topology.

This paper is organized as follows. In Section II, we propose a ring of coupled chaotic system. In Section III, 2- type chaos synchronization are shown. Be based on Section III, another example with VDP model are explained in Section IV. Finally, Section V concludes this paper.

\section{SYSTEM MODEL}

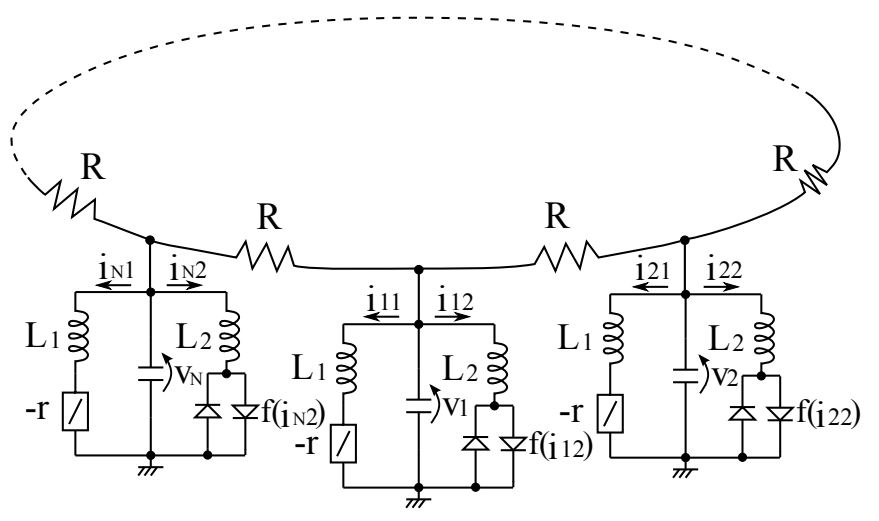

Fig. 1. System model.

The system model is shown in Fig. 1. Each subcircuit is coupled by resistor. Subcircuit is chaotic circuit which consists of three memory elements, one linear negative resistor, and bidirectionally-coupled diodes proposed by Nishio et al [5]. First, we approximate $i-v$ characteristic of bi-directionally-coupled diodes as following function:

$$
f\left(i_{n 2}\right)=\frac{1}{2}\left(\left|r_{d} i_{n 2}+V\right|-\left|r_{d} i_{n 2}-V\right|\right)
$$

where parameter $r_{d}$ denotes slope of nonlinear resistance. Then the system equation is described by the following $3 \times N$ dimensional ordinary differential equation:

$$
\left\{\begin{aligned}
L_{1} \frac{d i_{n 1}}{d t} & =v_{n}+r i_{n 1} \\
L_{2} \frac{d i_{n 2}}{d t} & =v_{n}-f\left(i_{n 2}\right) \\
C \frac{d v_{n}}{d t} & =-i_{n 1}-i_{n 2}+\frac{1}{R}\left(v_{n-1}-2 v_{n}+v_{n+1}\right) .
\end{aligned}\right.
$$


By changing the parameters and variables,

$$
\begin{aligned}
& t=\sqrt{L_{1} C} \tau, i_{n 1}=V \sqrt{\frac{C}{L_{1}}} x_{n}, i_{n 2}=V \sqrt{\frac{C}{L_{1}}} y_{n}, \\
& v_{n}=V z_{n}, \cdots=\frac{d}{d \tau}, \alpha=r \sqrt{\frac{C}{L_{1}}}, \beta=\frac{L_{1}}{L_{2}} \\
& \gamma=r_{d} \sqrt{\frac{C}{L_{1}}} \text { and } \delta=\frac{1}{R} \sqrt{\frac{L_{1}}{C}}
\end{aligned}
$$

the normalized system equation is described as follows:

$$
\left\{\begin{aligned}
\dot{x}_{n}= & \alpha x_{n}+z_{n}, \\
\dot{y}_{n}= & \beta\left\{z_{n}-\hat{f}\left(y_{n}\right)\right\}, \\
\dot{z}_{n}= & -x_{n}-y_{n} \\
& +\delta\left(z_{n-1}-2 z_{n}+z_{n+1}\right), \\
& (n=1,2, \cdots, N)
\end{aligned}\right.
$$

where

$$
\begin{aligned}
& z_{0}=z_{N}, \quad z_{N+1}=z_{1} \text { and } \\
& \quad \hat{f}\left(y_{n}\right)=\frac{1}{2}\left(\left|\gamma y_{n}+1\right|-\left|\gamma y_{n}-1\right|\right) .
\end{aligned}
$$

Figure 2 shows an example of asymmetric chaos attractor depending on initial value obtained from one subcircuit. For the computer simulation, we fixed the parameters as $\beta=3.0$ and $\gamma=470.0$. Asymmetric attractors are distinguished with 2 colors in simulation result. In this study, we distinguish the attractors as follows:

(1) A Poincaré section is defined at $z_{n}=1.0$ and $\dot{y}_{n}<0$.

(2) When the solution hits the Poincaré section, and $y_{n}>0.675$, the color is set as red and such state is defined state $\{M\}$. In the case of $y_{n} \leq 0.675$, the color is set as blue and defined state $\{P\}$.

This definition is important to grasp the domain of attractor in each subcircuit and as such, is being applied to following simulation results in this paper.

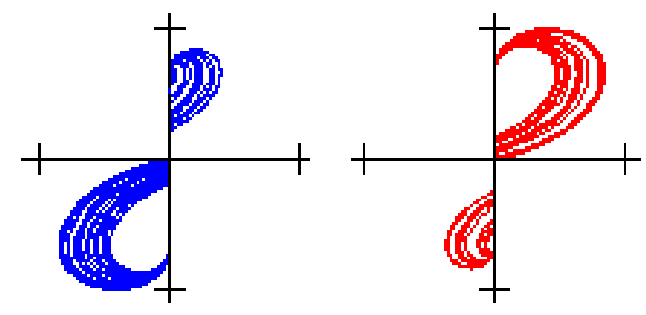

(a) $\alpha=0.35$
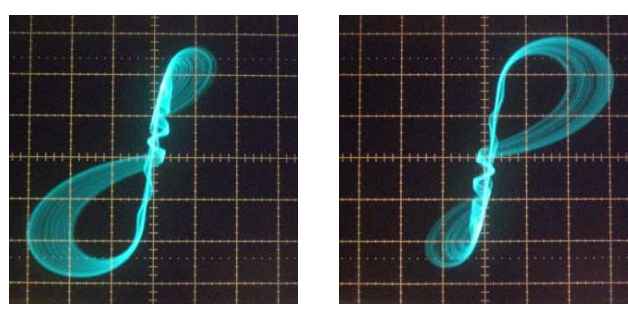

(b) $r=4.99$

Fig. 2. Asymmetric chaos attractor observed in a subcircuit. (a) Simulation result. Horizontal: $y_{1}$. Vertical: $z_{1} . \beta=3.0$ and $\gamma=470.0$. (b) Circuit experiment. Horizontal: $i_{12}\left[0.1 \mathrm{~mA} /\right.$ div]. Vertical: $v_{1}\left[\mathrm{~V} /\right.$ div]. $L_{1}=642.8[\mathrm{mH}]$, $L_{2}=211.1[\mathrm{mH}] C=7.06[\mathrm{nF}]$.

\section{2-TYPE CHAOS SYNCHRONIZATION}

2-type synchronization phenomena depending on initial values can be observed in the system shown in Fig. 1. Figure 3 shows the examples of synchronization phenomena. $N$-phase chaos synchronization is shown in Fig. 3 (a), and synchronization in Fig. 3 (b). Red and blue colors show the state $\{M\}$ and $\{P\}$ respectively. Both synchronization states are not synchronized rigorously since the system generates chaotic signal. However, those behaviours shown in Fig. 3 (a) and (b) are almost synchronized. Therefore these phenomena are called $N$-phase synchronization and synchronization respectively in this paper. It should be noted that when the subcircuits ( $n$ is odd number) take state $\{P\}$, while the others ( $n$ is even number) take state $\{M\}$ in Fig. 3. Namely, the subcircuits take opposite attractor state with respect to the neighbor subcircuits. An overview of attractor domain is shown in Fig. 4. This is the one of the characteristic of this chaotic circuit in case of coupled by resistor. It is considered that shifted bias waveform be caused due to the characteristic in this system. Figure 5 is

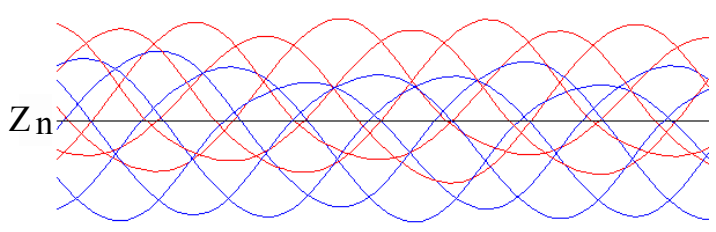

(a)

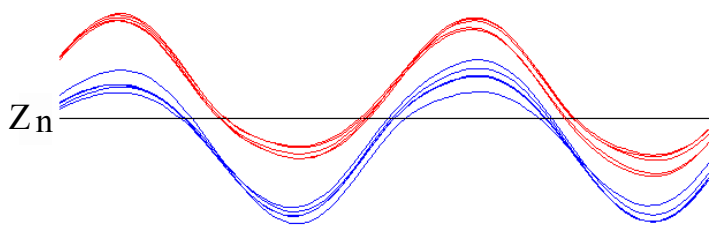

(b)

Fig. 3. 2-type synchronization phenomena depending on initial values. (a) $N$-phase chaos synchronization. (b) Synchronization. Vertical: $z_{n}$. Horizontal: $\tau$. $N=10, \alpha=0.425, \beta=3.0, \gamma=470.0$ and $\delta=0.20$.

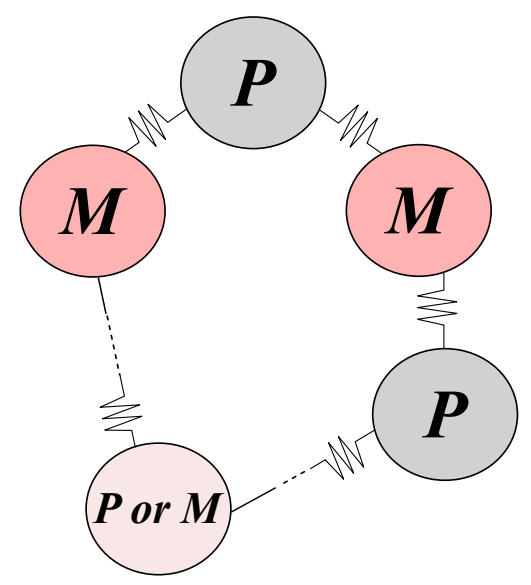

Fig. 4. Overview of attractor domain. 

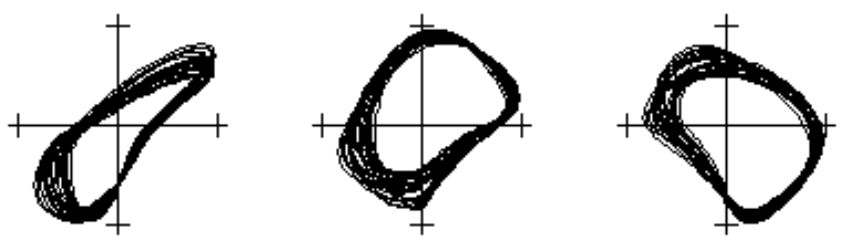

(a) $z_{1}$ vs $z_{2}$

(b) $z_{1}$ vs $z_{3}$
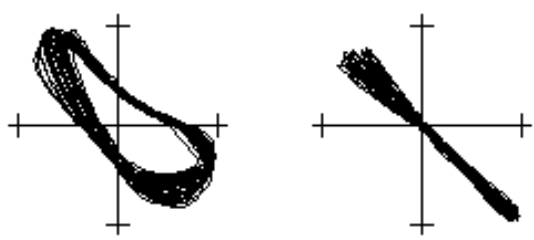

(e) $z_{1}$ vs $z_{6}$

(d) $z_{1}$ vs $z_{5}$

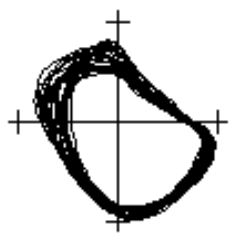

(g) $z_{1}$ vs $z_{8}$

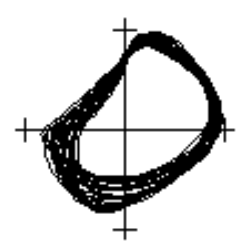

(h) $z_{1}$ vs $z_{9}$ (c) $z_{1}$ vs $z_{4}$

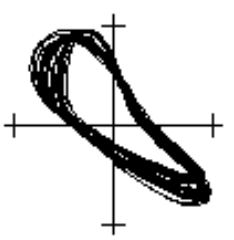

(f) $z_{1}$ vs $z_{7}$

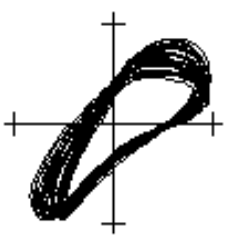

(i) $z_{1}$ vs $z_{10}$

Fig. 5. Lissajous figures in state of $N$-phase chaos synchronization (computer simulations). $z_{1}$ vs $z_{n} . \alpha=0.425, \beta=3.0, \gamma=470.0$ and $\delta=0.2$.

simulation results in state of $N$-phase chaos synchronization. The phase difference between $z_{1}$ and $z_{n}$ is enlarged from $z_{1}$ vs $z_{2}$ to $z_{1}$ vs $z_{6}$. Moreover, the subcircuits of diagonal positions are almost synchronized with anti-phase. This behaviour also can be observed in circuit experiment as shown in Fig. 6. Figure 7 shows time waveforms and Lissajous figure of $z_{1}$ and $z_{6}$ observed in simulation and circuit experiment. While $z_{1}$ takes state $\{P\}, z_{6}$ takes state $\{M\}$ and almost synchronized with anti-phase as shown in Fig. 7. Figure 8 shows another type synchronization. Although $z_{1}$ and $z_{6}$ take opposite attractor state, these circuits are almost synchronized in-phase. This behaviour can be observed by giving different initial values.

From this result, we can confirm that subcircuits which are located in diagonal position are synchronized with anti-phase or in-phase though both subcircuits take opposite attractor state. Moreover, 2-type synchronization phenomena can be observed in large-scale case around $N=50$.

\section{ANOTHER EXAMPLE}

From Section III, we found that two-type synchronization state is caused by ring topology and shifted bias waveforms. Since asymmetric attractors can be regarded as alternately shifted biases. Therefore we propose new subcircuit model with VDP circuit which includes ideal current source in order to confirm the essential factors of 2-type synchronization. The circuit model is shown in Fig. 9. Ideal current sources are used at the subcircuit located at $n$ (odd number) with forward direction, and the subcircuit located at $k$ (even number) with backward direction to make biases shift. We approximate the
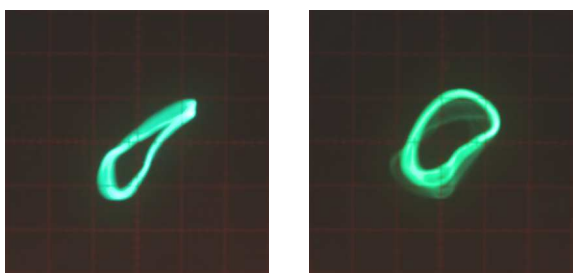

(b) $v_{1}$ vs $v_{3}$

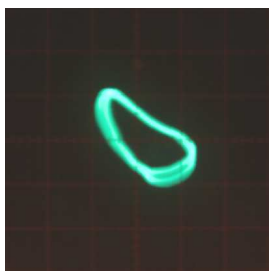

(d) $v_{1}$ vs $v_{5}$

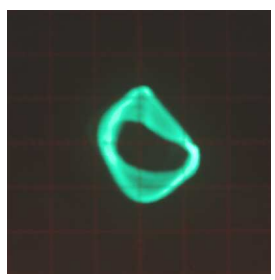

(g) $v_{1}$ vs $v_{8}$

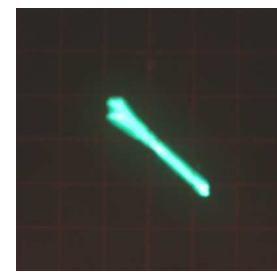

(e) $v_{1}$ vs $v_{6}$

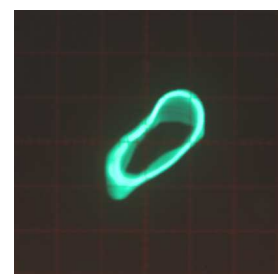

(h) $v_{1}$ vs $v_{9}$

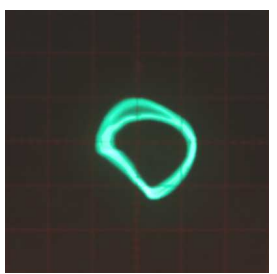

(c) $v_{1}$ vs $v_{4}$

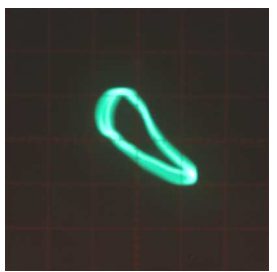

(f) $v_{1}$ vs $v_{7}$

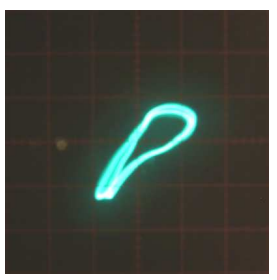

(i) $v_{1} \mathrm{VS} v_{10}$
Fig. 6. Lissajous figures in state of $N$-phase chaos synchronization (circuit experiments). $v_{1}$ vs $v_{n}[10 \mathrm{~V} / \mathrm{div}] . L_{1}=625[\mathrm{mH}] \pm 1 \%, L_{2}=209[\mathrm{mH}] \pm$ $1 \%, C=1.49[\mathrm{nF}] \pm 4 \%$ and $R=100.0[\mathrm{k} \Omega] \pm 1 \%$. (a)
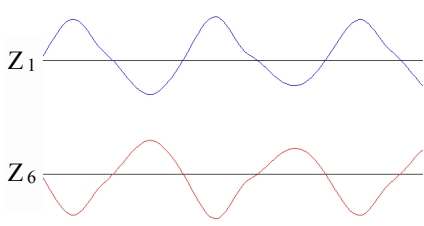

(b)

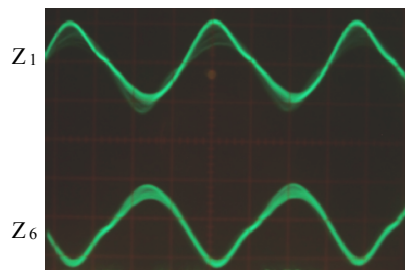

(1)
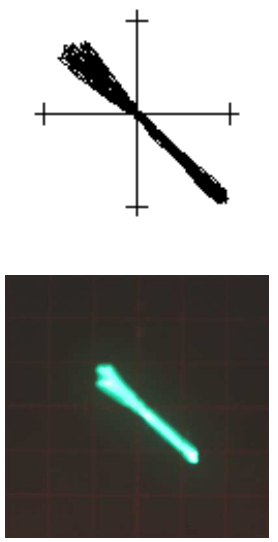

(2)
Fig. 7. Almost anti-phase synchronization state for $N=10$. (a) Simulation result. (1) Time waveforms of $z_{1}$ and $z_{6}$. (2) $z_{1}$ vs $z_{6}$. (parameter values are the same as those in Fig. 5). (b) Circuit experiment. (1) Time waveforms of $v_{1}$ and $v_{6}$. (2) $v_{1}$ vs $v_{6}$. (parameter values are the same as those in Fig. 6).

$v_{n}-i_{R n}$ characteristics of nonlinear resistor including ideal current source as follows:

$$
i_{R n}=-g_{1} v_{n}+g_{3} v_{n}^{3}+(-1)^{n} J
$$


(a)
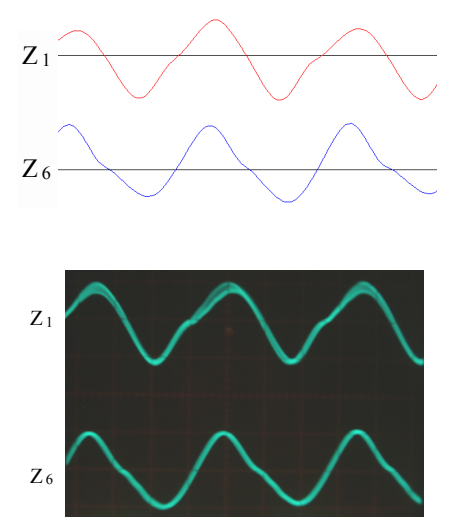

(1)
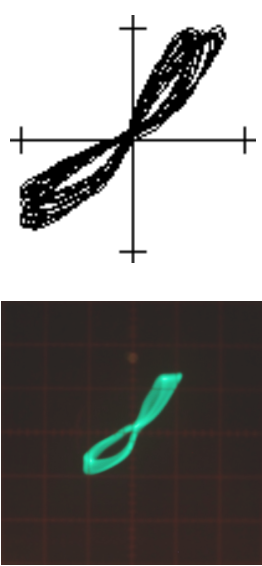

(2)

Fig. 8. Synchronization state for $N=10$. (a) Simulation result. (1) Time waveforms of $z_{1}$ and $z_{6}$. (2) $z_{1}$ vs $z_{6}$. (parameter values are the same as those in Fig. 5). (b) Circuit experiment. (1) Time waveforms of $v_{1}$ and $v_{6}$. (2) $v_{1}$ vs $v_{6}$. (parameter values are the same as those in Fig. 6).

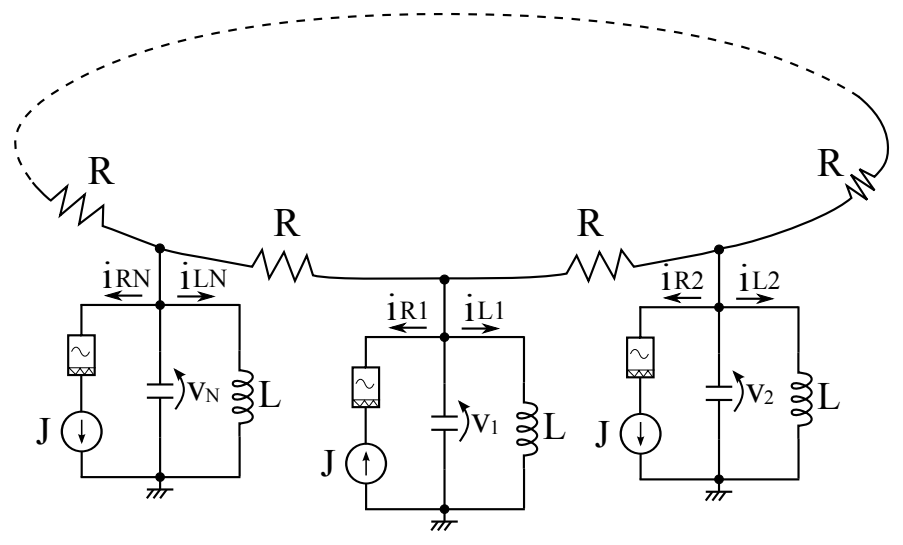

Fig. 9. System model with VDP-model.

By changing the parameters and variables,

$$
\begin{aligned}
& t=\sqrt{L C} \tau, v_{n}=\sqrt{\frac{g_{1}}{g_{3}}} x_{n}, i_{L n}=\sqrt{\frac{g_{1}}{g_{3}}} \sqrt{\frac{C}{L}} y_{n}, \\
& \varepsilon=g_{1} \sqrt{\frac{L}{C}}, \delta=\frac{1}{R} \sqrt{\frac{L}{C}} \text { and } \beta=\sqrt{\frac{g_{3}}{g_{1}}} \sqrt{\frac{L}{C}} J
\end{aligned}
$$

the normalized system equation is described as follows:

$$
\left\{\begin{aligned}
\dot{x}_{n}= & \epsilon\left(1-x_{n}^{2}\right) x_{n}-y_{n}+(-1)^{n} \beta \\
& +\delta\left(x_{n-1}-2 x_{n}+x_{n+1}\right) \\
\dot{y}_{n}= & x_{n}, \quad(n=1,2, \cdots, N)
\end{aligned}\right.
$$

where

$$
x_{0}=x_{N} \text { and } x_{N+1}=x_{1} .
$$

Figure 10 shows simulation results for the system shown in Fig. 9. 2-type synchronization depending on initial values also can be observed in this system. The time waveforms are described with 2 colors following condition:

(1) Blue: $n$ is odd.

(2) Red: $n$ is even.

(a)
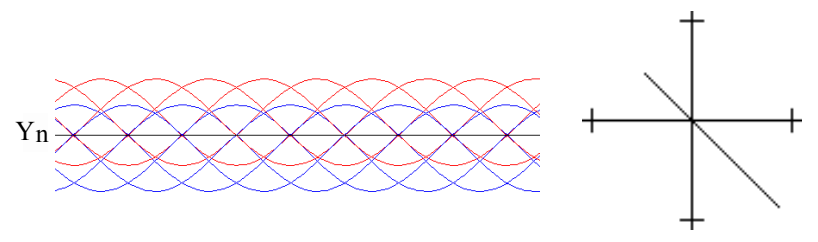

(b)

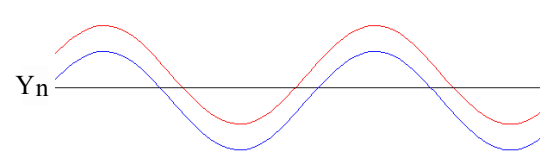

(1)

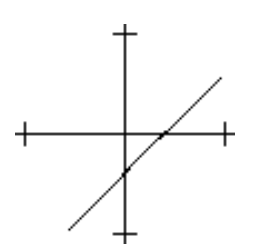

(2)
Fig. 10. 2-type synchronization phenomena observed in another system model as shown in Fig. 9. (a) $N$-phase synchronization state. (b) Synchronization state. (1) Time waveforms of $y_{n}$. (2) $y_{1}$ vs $y_{6} . N=10, \varepsilon=0.08, \beta=0.3$ and $\delta=0.05$.

In this case, each subcircuit exactly synchronized with $N$ phase. Moreover, the subcircuits located in diagonal position are synchronized with opposite phase shown in Fig. 10 (2). Those 2-type synchronization phenomena also can be observed around $N=50$.

From these results, we specified that the essential factors of 2-type synchronization are ring topology and alternately shifted biases.

\section{CONCLUSION}

In this study, we have investigated synchronization phenomena on coupled nonlinear circuit as a ring. As a result, $N$ phase synchronization and simple synchronization phenomena are observed in 2 system models. Moreover, it is declared that essential factors of 2-type synchronization depending on initial values are ring topology and alternately shifted bias. This result is extremely interesting and would give the effective suggestion to make clear the nonlinear phenomena in large-scale highdimensional system. In our future work, we will investigate the mechanism of $\mathrm{N}$-phase synchronization phenomena in the system with VDP model by using averaging method.

\section{REFERENCES}

[1] L.O. Chua, M. Komuro and T. Matsumoto, "The doulbe scroll family", IEEE Transactions on Circuit and Systems, vol. 33, no. 11, pp. 10731118, Oct. 1986.

[2] H. Sekiya, S. Mori and I. Sasase, "Synchronization of Self-Switching phenomena on Full-Coupled Chaotic Oscillators", IEICE Trans., vol.J83A, no. 11 pp. 1264-1275, 2000.

[3] T. Nishimoto, Y. Hosokawa and Y. Nishio, "Anti-Phase Synchronization of Switching Phenomena in Globally Coupled System of Chaotic Circuits", IEICE Technical Report, no. NLP-48 \& CAS2010-32, pp. 1-4, 2010.

[4] Y. Nishio, K. Suzuki, S. Mori and A. Ushida, "Synchronization in Mutually Coupled Chaotic Circuits", Proceedings of European Conference on Circuit Theory and Design(ECCTD'93), vol. 1, pp. 637-642, Aug. 1993.

[5] Y. Nishio, N. Inaba, S. Mori and T. Saito, "Rigorous Analyses of Windows in a Symmetric Circuit", IEEE Transactions on Circuit and Systems, vol. 37, no. 4, pp. 473-487, Apr. 1990. 